\title{
A Randomised Controlled Trial of Local Infiltration Analgesia Versus Femoral Nerve Block for Postoperative Analgesia Following Total Knee Arthroplasty
}

Yang Min Ng ${ }^{1}$, Fiona Martin ${ }^{1}$, Hugh B. Waterson 2 , Adam Green ${ }^{3}$, Jeremy Preece ${ }^{4}$, Nerida Robinson 5 Jon Phillips ${ }^{6}$, Keith S. Eyres ${ }^{6}$, Andrew D. Toms ${ }^{6}$, James Simpson ${ }^{1}$

1. Anaesthesiology, Royal Devon \& Exeter NHS Foundation Trust, Exeter, GBR 2. Orthopaedic Surgery, Royal Devon \& Exeter NHS Foundation Trust, Exeter, GBR 3. Anaesthesiology, University Hospitals Plymouth NHS Trust, Plymouth, GBR 4. Anaesthesiology, Northern Devon Healthcare NHS Trust, Barnstaple, GBR 5. Anaesthesiology, The Canberra Hospital, Canberra, AUS 6. Orthopaedics, Royal Devon \& Exeter NHS Foundation Trust, Exeter, GBR

Corresponding author: Hugh B. Waterson, benwaterson@nhs.net

\section{Abstract \\ Background}

Total knee replacement is often associated with significant postoperative pain. Although the use of a femoral nerve block is well-established, local infiltration analgesia has gained popularity in recent years. We compared single-shot local infiltration analgesia with a single-shot femoral nerve block for patients undergoing primary total knee arthroplasty.

\section{Methods}

A total of 194 patients were randomised to receive either local infiltration analgesia (150 $\mathrm{ml}$ bupivacaine $0.067 \%$ with adrenaline) or a femoral nerve block ( $20 \mathrm{ml} 0.375 \%$ levobupivacaine). Both groups received spinal anaesthesia. The primary outcome measure was the total morphine consumption. Secondary outcome measures included: post-operative pain scores, rehabilitation goals, readiness for discharge, and physical, mental, and functional outcomes, including the Oxford Knee Score (OKS).

\section{Results}

A total of 69 patients in the local infiltration analgesia group and 79 patients in the femoral nerve block group were analysed. Median total morphine consumption was significantly greater in the local infiltration analgesia group as compared to the femoral nerve block group ( $54.67 \mathrm{mg}$ vs $45 \mathrm{mg}$, respectively, $\mathrm{p}=0.0388$ ). The post-operative OKS at six weeks was slightly more improved for the femoral nerve block group than for local infiltration analgesia (12.5 vs 9 point median improvements for the femoral nerve block and local infiltration analgesia groups, respectively, $\mathrm{p}=0.0261$ ). There were no statistically significant differences in other secondary outcome measures.

Received 08/19/2020

Review began 08/19/2020 Review ended 08/26/2020 Published 09/02/2020

(c) Copyright 2020 $\mathrm{Ng}$ et al. This is an open access article distributed under the terms of the Creative Commons Attribution License CC-BY 4.0., which permits unrestricted use, distribution, and reproduction in any medium, provided the original author and source are credited.

\section{Conclusion}

A single-shot femoral nerve block significantly reduces the opioid requirement for primary total knee arthroplasty but is otherwise comparable to single-shot local infiltration analgesia.

Categories: Anesthesiology, Orthopedics

Keywords: total knee arthroplasty, femoral nerve, local anaesthetic

\section{Introduction}

Total knee arthroplasty (TKA) is often associated with significant early post-operative pain. There is an inherent conflict between the need to rest the operated limb to limit the pain and the need to move it to avoid stiffness, facilitating a timely recovery [1]. A femoral nerve block (FNB) has long been advocated as an effective part of the post-operative analgesia regime for TKR [2]. In recent years, however, local infiltration analgesia (LIA) has gained popularity, whereby surgeons inject a relatively large volume of a dilute local anaesthetic directly into and around the operative site. LIA has since been embraced at our centre, with potential advantages over other methods, including speed, simplicity, and reduced postoperative weakness.

Multiple studies have compared LIA with other regional techniques, but many of these looked at catheterbased techniques rather than the single-shot technique [3-5]. Our local experience has found that catheter techniques are time-consuming in the theatre and can be difficult to manage in the ward. There are also concerns about the increased risk of post-operative infections, which can be catastrophic [6]. At the start of our study in 2015, few trials directly compared single-shot techniques. 
We, therefore, conducted a single-centre, randomised controlled trial of single-shot LIA versus single-shot FNB for postoperative analgesia following TKA.

\section{Materials And Methods}

Ethical approval was granted by the South West (Exeter) Research Ethics Service Committee. The trial was conducted at the Royal Devon \& Exeter NHS Foundation Trust in the UK and prospectively registered with ClinicalTrials.gov (Identifier: NCT0228892).

We screened and recruited patients undergoing primary TKA from the pre-operative assessment clinic. We excluded patients having TKA for trauma and unicompartmental and bilateral surgery, patients with contraindications to spinal anaesthesia or peripheral nerve blocks, allergy to local anaesthetics or morphine, and problems with communication that would have compromised informed consent. We also excluded patients under active management by chronic pain services, patients with chronic strong opioid use (e.g. morphine, oxycodone, buprenorphine and methadone), or patients taking neuropathic analgesic agents (e.g. gabapentin, pregabalin, or amitriptyline). Pre-operative physical and mental health and functional status were assessed using the Oxford Knee Score (OKS), the EuroQol 5 Dimension Score (EQ-5D-5L), and the Hospital Anxiety and Depression Scale (HADS).

Based on a previous study conducted at our hospital [7], we expected patients in the FNB group to use a median of $44 \mathrm{mg}$ morphine in total over 72 hours. In order to make our results as robust as possible, we set an effect size of 0.4 , making a difference in total morphine consumption between the two groups of $>40 \%$ as clinically significant. With a significance level of $<0.05$ and power of $80 \%$, we calculated that at least 64 patients per group would be required.

We used block randomisation with numbered, sealed envelopes, which informed clinicians of the relevant protocol shortly before surgery. It was considered neither practical nor safe to blind the healthcare staff directly involved with patient care to the treatment arm of the study. Those collecting, reviewing, and analyzing the data were, however, blinded and patients were not informed of their allocation arm.

All patients received a spinal anaesthetic containing 2.5-3.5 $\mathrm{ml} 0.5 \%$ plain levobupivacaine. Additional sedation or general anaesthesia (including intravenous fentanyl up to $2 \mu \mathrm{g} / \mathrm{kg}$ ) was administered at the discretion of the anaesthetist. All patients received the same type of total knee arthroplasty performed with a standard technique without the use of a tourniquet.

Patients in the LIA group received a single shot of $40 \mathrm{ml}$ 0.25\% bupivacaine with adrenaline 1:200,000, diluted with $110 \mathrm{ml}$ of $0.9 \%$ saline, making a total volume of $150 \mathrm{ml}$. This was divided into thirds and administered by the surgeon towards the end of the operation: $50 \mathrm{ml}$ was injected into the posterior capsule before cementing, $50 \mathrm{ml}$ into the medial and lateral capsules, and $50 \mathrm{ml}$ into subcutaneous tissues and in and around the vastus medialis and sartorius muscles.

Patients in the FNB group received a single-shot femoral nerve block before the operation using $20 \mathrm{ml}$ $0.375 \%$ levobupivacaine, guided by ultrasound or a peripheral nerve stimulator.

Postoperatively, all patients received morphine patient-controlled analgesia (PCA) pump and regular paracetamol and ibuprofen (unless contraindicated or already on an alternative non-steroidal antiinflammatory drug). Oral morphine was prescribed for all patients following the discontinuation of their PCA, usually on postoperative day 1 .

The primary outcome measure was total morphine consumption during the first 72 hours postoperatively, measured during the following four time periods: 0-12 hours, 12-24 hours, 24-48 hours, and 48-72 hours. This was expressed as the intravenous equivalent dose of morphine (i.e., the total intravenous dose via boluses and the patient's PCA, plus one-third of any oral morphine administered).

Secondary outcome measures were: postoperative pain scores using the Numerical Rating Scale (NRS); rehabilitation goals (stand and sit out by postoperative day 1, walk to the bathroom by postoperative day 2 , walk independently with crutches by postoperative day 4); medical fitness for discharge; Quality of Recovery 40 (QoR-40) questionnaire; Oxford Knee Score (OKS) and EuroQol 5 Dimensions Score (EQ-5D-5L), both collected preoperatively and six weeks postoperatively.

Statistical analysis was undertaken using IBM SPSS Statistics for Windows v20.0 (IBM Corp., Armonk, NY). Shapiro-Wilk was used to test for parametricity. Where parametric data were identified, means and standard deviations were reported. Where non-parametric data were identified, medians and interquartile ranges were reported. For statistical significance, we used the Mann-Whitney U test for median total morphine consumption, OKS, 5Q-5D-5L scores, QoR scores, and readiness for discharge; the student's unpaired t-test for pain scores, and the chi-squared test for complications. We considered $\mathrm{p}<0.05$ to be statistically significant. 


\section{Cureus}

\section{Results}

The study was conducted between March 2015 and July 2018. We recruited a total of 200 patients (Figure 1). Six were excluded and the remaining 194 were randomised. Of these, 25 patients did not receive the allocated intervention so were withdrawn (14 in LIA and 11 in FNB groups, respectively). Of note, in the LIA group, three patients were deemed inappropriate to receive LIA alone and, therefore, FNB was administered.

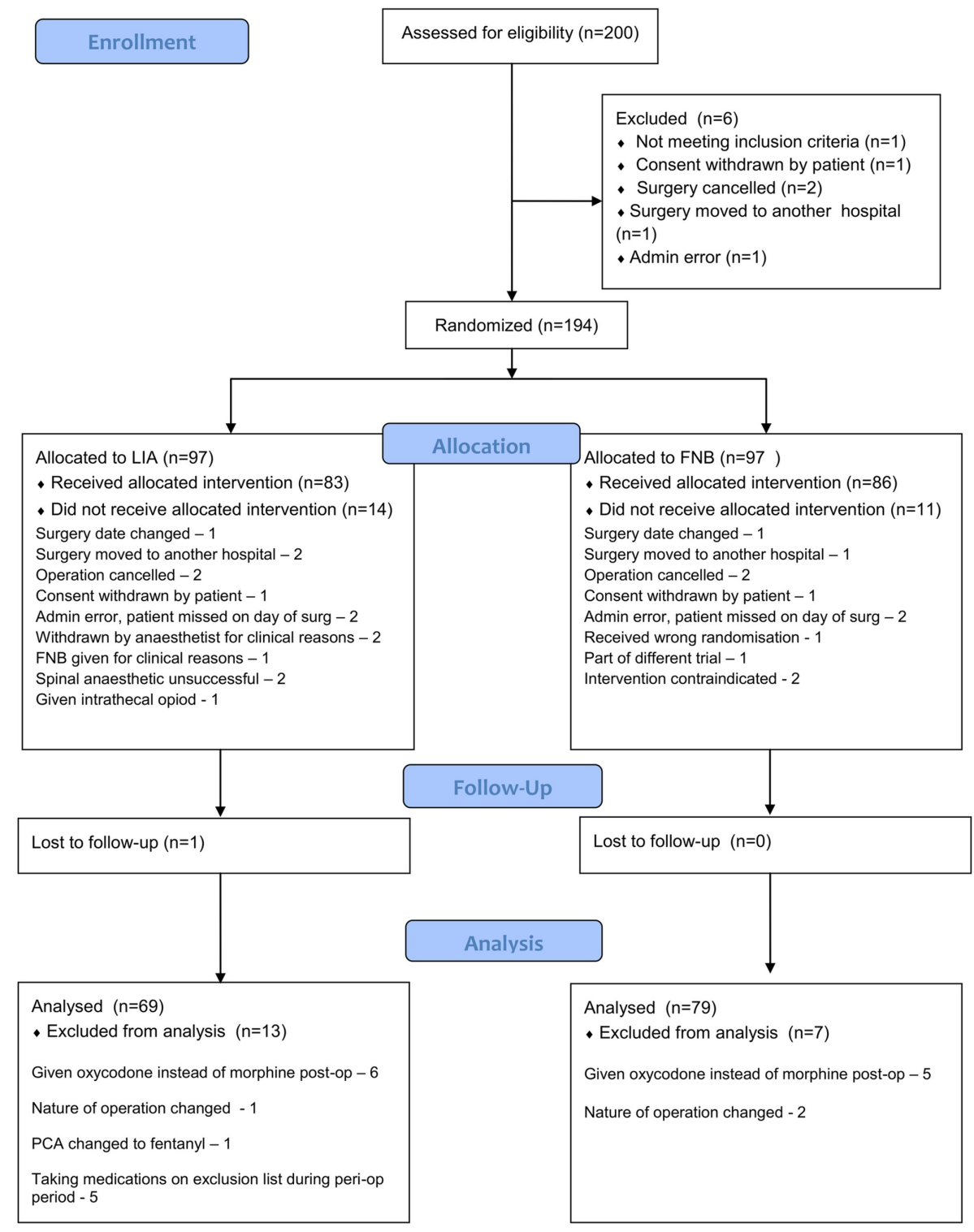

FIGURE 1: CONSORT diagram of patient recruitment

One patient in the LIA group was lost to follow-up, and 20 patients were excluded from the analysis due to protocol violations (13 in LIA and 7 in FNB groups, respectively). Of these, a total of 12 patients across both groups were excluded, as they were prescribed non-morphine analgesics postoperatively. This included 11 patients who were given oxycodone, and one patient in the LIA group was prescribed fentanyl PCA. The final analysis was performed on an 'as treated' basis. Following exclusions, we analysed 148 patients.

Baseline characteristics, including gender, OKS, and EQ-5D-5L scores, were well-matched and comparable in both intervention groups (Table 1) ( $p>0.05$ for all preoperative scores). Ninety-seven point six (97.6\%) of patients were Caucasian British. The proportions of both groups having operations in the morning (LIA 35; FNB 35), the afternoon (LIA 42; FNB 50), and postoperative care in each hospital ward were not significantly different. The majority of operations ( $\mathrm{n}=145,98 \%$ ) were conducted by two surgeons. Three anaesthetists performed the most ( $\mathrm{n}=62,41.8 \%$ ), but 17 anaesthetists were involved in only one operation. 


\section{Cureus}

\begin{tabular}{|c|c|c|}
\hline & LIA $(n=69)$ & FNB(n=79) \\
\hline Gender, $n, M / F$ & 38/31 & 40/39 \\
\hline Pre-EQ-5D-5L Total Score, mean (SD) & $12.48(3.2)$ & $13.17(2.66)$ \\
\hline Pre-OKS, mean (SD) & $39.63(7.48)$ & $40.17(6.82)$ \\
\hline Pre-HAD Total Score, mean (SD) & $8.94(5.1)$ & $10.63(6.34)$ \\
\hline
\end{tabular}

\section{TABLE 1: Baseline measures pre-intervention}

EQ-5D-5L = 5-level EuroQol 5 Dimension Score; OKS = Oxford Knee Score; HAD = Hospital Anxiety \& Depression Scale; SD = Standard Deviation

\section{Primary outcome measure}

The median total morphine consumption was significantly greater in the LIA group than in the FNB group ( $54.67 \mathrm{mg}$ and $45 \mathrm{mg}$, respectively, $\mathrm{p}=0.0388$, Table 2 ), a median difference of $10.67 \mathrm{mg}$.

\begin{tabular}{|c|c|c|c|}
\hline & LIA $(n=69)$ & FNB $(n=79)$ & p-value \\
\hline Recovery & $0(0-1)$ & $0(0-0)$ & \\
\hline $0-12$ hours & $19.5(11-29)$ & $13(6.8-19.0)$ & \\
\hline 13-24 hours & $4.5(1-12)$ & $2(0.5-6)$ & \\
\hline 25-48 hours & $15.67(6.7-27)$ & $14(6.8-25)$ & \\
\hline 49-72 hours & $4.67(0-12)$ & $6.67(0-13.3)$ & \\
\hline Total Morphine consumption & $54.67(28-85)$ & $45(24.2-62.3)$ & 0.0388 \\
\hline
\end{tabular}

\section{TABLE 2: Postoperative morphine consumption expressed in $\mathrm{mg}$, intravenous dose equivalent}

Figures expressed as median values and interquartile range

\section{Secondary outcome measures}

Pain Scores

Mean pain scores were negligible or zero in recovery, peaked at 12 hours (LIA group) and postoperative day 2 (FNB group), before decreasing on postoperative day 3 in both groups (Table 3). A split plot was used to look for repeated-measure and between-groups effects (Figure 2). However, the differences in overall pain score were not statistically significant $(\mathrm{p}=0.3129)$. 


\section{Cureus}

\begin{tabular}{|c|c|c|c|}
\hline & LIA $(n=69)$ & FNB $(n=79)$ & p-value \\
\hline Pain score in recovery & $0.2(0-0.452)$ & $0(0-0)$ & \\
\hline Pain score at 3 hours & $2(1.29-2.71)$ & $1.18(0.675-1.69)$ & \\
\hline Pain score at 6 hours & $3.46(2.75-4.17)$ & $2.85(2.24-3.46)$ & \\
\hline Pain score at 12 hours & $3.79(3.79-2.61)$ & $2.53(1.95-3.11)$ & \\
\hline Pain score at 24 hours & $3.72(3.13-4.31)$ & $3.24(2.7-3.78)$ & \\
\hline Pain score at postop Day 2 & $3.37(2.73-4.01)$ & $3.6(3.06-4.14)$ & \\
\hline Pain score at postop Day 3 & $1.91(1.36-2.46)$ & $2.91(2.32-3.5)$ & \\
\hline Overall pain score in the first 72 hours & $2.50(2.19-2.80)$ & $2.29(2.03-2.56)$ & 0.3129 \\
\hline
\end{tabular}

TABLE 3: Postoperative mean pain scores scaled from $0-10$, with 0 being no pain and 10 being the worst pain ever experienced

Figures expressed as mean values and $95 \%$ confidence interval.

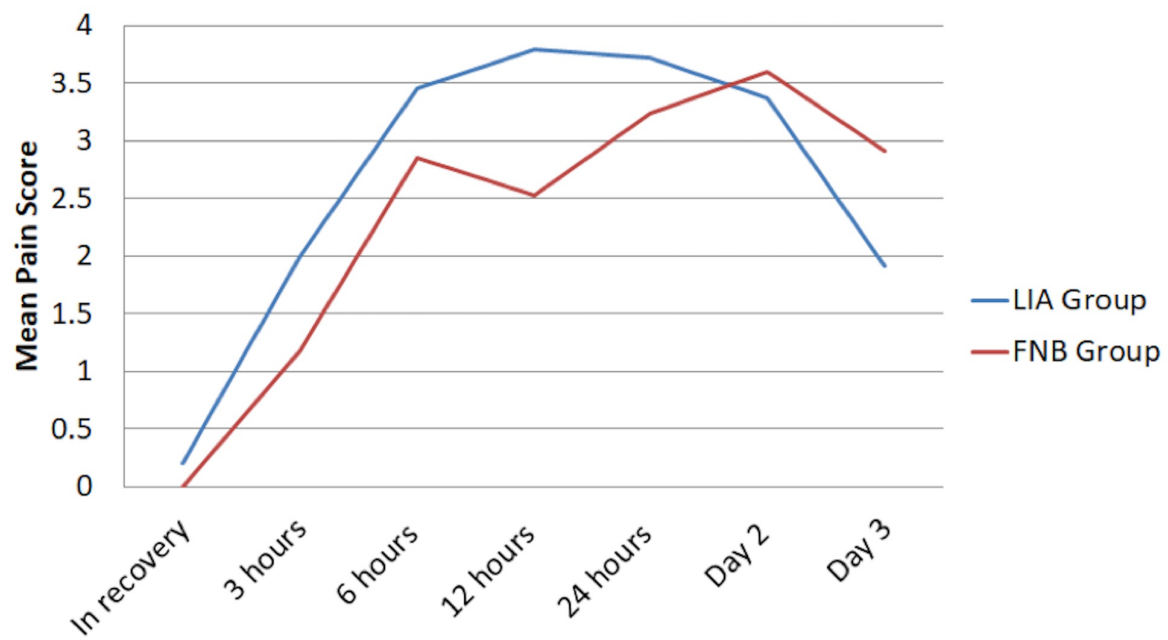

FIGURE 2: Postoperative pain scores at different time intervals postoperatively scaled from $0-10$, with 0 being no pain and 10 being the worst pain ever experienced

Figures expressed as mean values.

Achievement of Rehabilitation Goals

A higher proportion of patients in the FNB group achieved rehabilitation goals at postoperative day 1, but the differences were less apparent after that and did not reach significance at any stage $(\mathrm{p}=0.145$, Table 4$)$. 


\section{Cureus}

\begin{tabular}{|c|c|c|c|}
\hline & LIA & FNB & P-value \\
\hline Postop Day 1 & $53 / 69(76.8 \%)$ & $68 / 79(86.1 \%)$ & \\
\hline Postop Day 2 & $53 / 69(76.8 \%)$ & $63 / 79(79.5 \%)$ & \\
\hline Postop Day 4 & $62 / 39(89.9 \%)$ & $67 / 79(85.1 \%)$ & \\
\hline Ready to discharge (median) & Day 3 & Day 4 & 0.2941 \\
\hline
\end{tabular}

TABLE 4: Proportion of patients reaching rehabilitation goals, whereby patients are expected to stand and sit out by end of postop day 1 , able to walk to the bathroom by end of day 2 , and walking independently with crutches by end of day 4

Readiness for Discharge

There was no significant difference in time to discharge between the two groups. The median number of days was 4 (interquartile range 3-5) and 3 (interquartile range 3-4) for the FNB and LIA groups, respectively $(\mathrm{p}=0.2941)$.

Patient Recovery, Satisfaction, and Functional Improvement

Postoperative Quality of Recovery 40 (QoR-40) and 5-level EuroQol 5 Dimension Score (EQ-5D-5L) scores were not significantly different between the groups (Table 5). There was, however, a statistically significant difference in the improvement of the Oxford Knee Score six weeks postoperatively, with a median improvement of 12.5 points in the FNB group vs 9 in the LIA group ( $\mathrm{p}=0.0261)$.

\begin{tabular}{|l|l|l|}
\hline & LIA (n=69) & FNB (n=79) \\
\hline QoR-40 total score (day 2 postop) & $175(162-181)$ & $176(154-187)$ \\
\hline EQ-5D-5L total score (6 weeks postop) & $9(7-10)$ & $9(6-11)$ \\
\hline Improvement in EQ-5D-5L total score & 3 & 5 \\
\hline OKS total score (6 weeks postop) & $27(22-33)$ & $26(20-35)$ \\
\hline Improvement in OKS score & 9 & 12.5 \\
\hline
\end{tabular}

TABLE 5: Postoperative physical, mental and functional status questionnaire scores

QoR-40 = Quality of Recovery 40 Questionnaire; EQ-5D-5L = 5-level EuroQol 5 Dimension Score; OKS = Oxford Knee Score. Figures expressed as median scores and (interquartile range)

\section{Complications}

There was one death in the LIA group. The patient developed multiorgan failure and subsequently died. There was a greater number of major and minor complications in the FNB Group $(17 / 79,21.52 \%)$ than in the LIA group (10/69, 14.49\%), but the difference was not statistically significant (Risk Ratio 1.4, 95\% CI: 0.69 to 2.86, $\mathrm{p}=0.2695$ ) (Table 6). The largest percentage of minor complications were wound site infections, and these cannot be attributed to the FNB. 


\section{Cureus}

\begin{tabular}{|c|c|}
\hline LIA $(n=69)$ & FNB $(n=79)$ \\
\hline Major Complications & Major Complications \\
\hline \multirow[t]{2}{*}{ Death - 1 (1.4\%) } & Pneumonia - 1 (1.3\%) \\
\hline & Myocardial infarction - 1 (1.3\%) \\
\hline Minor Complications & Minor Complications \\
\hline Minor wound site infection - 2 (2.9\%) & Minor wound site infection - 7 (8.9\%) \\
\hline Fall - 1 (1.4\%) & Fall - $1(1.3 \%)$ \\
\hline Urinary retention $-2(2.9 \%)$ & Delirium - 2 (2.5\%) \\
\hline Acute renal impairment - 1 (1.4\%) & Urinary tract infection $-2(2.5 \%)$ \\
\hline \multirow[t]{3}{*}{ Other complications - 3 (4.3\%) } & Cardiac dysrhythmia -1 (1.3\%) \\
\hline & Pain and poor rehabilitation 1 (1.3\%) \\
\hline & Locked knee 1 (1.3\%) \\
\hline
\end{tabular}

\section{TABLE 6: Major and minor complications}

Figures expressed as $\mathrm{n}(\%)$

\section{Discussion}

Our study demonstrated that patients in the FNB group used significantly less morphine postoperatively than the LIA group. The difference was particularly apparent during the first 24 hours. Whilst there was no significant difference between pain scores, given that there was rescue analgesia immediately available during the early postoperative period in the form of patient-controlled analgesia, we expect that those in more pain simply used more morphine.

We also noted a slightly better improvement in the postoperative Oxford Knee Score for the FNB group. This may indicate that the FNB group had subjectively less pain and better function at six weeks postoperatively when compared to the LIA group. However, the clinical relevance of this small numerical difference is unclear, and the difference between the groups (12.5 points in the FNB group vs 9 in the LIA group) was smaller than the minimal clinically important difference for the Oxford Knee Score [8].

There was one postoperative fall in each group, but for the one in the FNB group, the non-operative leg was at fault. There were no falls relating to weakness from FNB. However, given how catastrophic a fall may be, adductor canal blocks have gained traction in recent years, as they relatively spare quadriceps strength when compared to FNB [9]. Given the elderly and frequently comorbid population presenting for TKA surgery, the other complications highlighted in Table 6 were not considered to be unusual or likely to have been related to the treatment interventions of the study.

The FNB group appeared to achieve readiness for discharge one day later than the LIA group. However, this difference was not statistically significant.

There were several potential limitations to our study. The total masses of bupivacaine and levobupivacaine used were different between the groups (100 mg vs $75 \mathrm{mg}$ for the LIA and FNB groups, respectively). This was because the doses of bupivacaine and levobupivacaine for both interventions were standard at our institution at the time of the study. The LIA mixture was comparable to others in both constituents and volume [10]. Twenty $\mathrm{ml}$ of $0.375 \%$ levobupivacaine for the FNB was chosen to strike a balance between adequate analgesia and prolonged motor blockade, which may adversely affect mobilisation and rehabilitation. It, therefore, follows that the true differences between FNB and LIA may be even greater than demonstrated by our study.

Other limitations include the number of different anaesthetists involved and that some patients received a general anaesthetic in addition to their spinal anaesthetic. We allowed individual anaesthetists to utilise either ultrasound or nerve stimulator as per their usual practise to administer femoral nerve blockade. There 
was a need to balance a rigid protocol with real-world flexibility to facilitate service delivery, minimise protocol violations, and complete recruitment within a reasonable timeframe. Our study was thus designed to reflect a reasonable compromise, which was felt unlikely to have a significantly detrimental effect on the validity of our results.

Following the completion of our trial, several studies and meta-analyses have since been published comparing LIA with FNB [11-18]. Our trial supported many of the findings of these studies, including the lack of differences in postoperative pain scores, rates of complication, and length of hospital stay. However, our trial highlighted a significant difference in morphine consumption, with less morphine use in the FNB group. Our trial was also unique in identifying slightly better improvement in long-term functional outcome for patients who have received femoral nerve blockade, although the clinical relevance of this is unclear.

\section{Conclusions}

We conclude that single-shot LIA is comparable to single-shot FNB for primary total knee arthroplasty. However, FNB significantly reduces the opioid requirement. FNB, therefore, remains an important tool and should be considered especially in patients where analgesic strategies may be challenging.

\section{Additional Information \\ Disclosures}

Human subjects: Consent was obtained by all participants in this study. The South West (Exeter) Research Ethics Service Committee issued approval NCT02288923. Ethical approval was granted by the South West (Exeter) Research Ethics Service Committee. The trial was conducted at the Royal Devon \& Exeter NHS Foundation Trust in the UK, and registered with ClinicalTrials.gov (Identifier: NCT02288923). Animal subjects: All authors have confirmed that this study did not involve animal subjects or tissue. Conflicts of interest: In compliance with the ICMJE uniform disclosure form, all authors declare the following: Payment/services info: All authors have declared that no financial support was received from any organization for the submitted work. Financial relationships: All authors have declared that they have no financial relationships at present or within the previous three years with any organizations that might have an interest in the submitted work. Other relationships: All authors have declared that there are no other relationships or activities that could appear to have influenced the submitted work.

\section{Acknowledgements}

The authors would like to thank Dr Roy Powell (Statistical Advisor, RDENFT; Honorary Senior Lecturer, University of Exeter Medical School); Professor Gordon Taylor (Professor of Medical Statistics, University of Exeter Medical School); Dr Mark Rockett (Consultant Anaesthetist, University Hospitals Plymouth NHS Trust); and Mr Vipul Mandalia (Consultant Orthopaedic Surgeon, RDENFT).

\section{References}

1. Borgeat A: The role of regional anesthesia in patient outcome: orthopedic surgery. Tech Reg Anesth Pain Manag. 2008, 12:178-182. 10.1053/i.trap.2008.09.007

2. Fischer H, Simanski C, Sharp C, et al.: A procedure-specific systematic review and consensus recommendations for postoperative analgesia following total knee arthroplasty. Anaesthesia. 2008, 63:1105-1123. 10.1111/j.1365-2044.2008.05565.x

3. Toftdahl K, Nikolajsen L, Haraldsted V, Madsen F, Tnnesen EK, Sballe K: Comparison of peri- and intraarticular analgesia with femoral nerve block after total knee arthroplasty. Acta Orthop. 2007, 78:172179. 10.1080/17453670710013645

4. Carli F, Clemente A, Asenjo JF, et al.: Analgesia and functional outcome after total knee arthroplasty: periarticular infiltration vs continuous femoral nerve block. Br J Anaesth. 2010, 105:185-195. 10.1093/bja/aeq112

5. Affas F, Nygårds E, Stiller C, Wretenberg P, Olofsson C: Pain control after total knee arthroplasty: a randomized trial comparing local infiltration anesthesia and continuous femoral block. Acta Orthop. 2011, 82:441-447. 10.3109/17453674.2011.581264

6. Kehlet $\mathrm{H}$, Anderson L: Local infiltration analgesia in joint replacement: the evidence and recommendations for clinical practice. Acta Anaesthesiol Scand. 2011, 55:778-784. 10.1111/j.1399-6576.2011.02429.x

7. Looseley A, Pappin D, Knight T, et al.: A randomised, observer blinded, controlled trial of intrathecal diamorphine versus femoral nerve block for post-operative analgesia following primary total knee arthroplasty. Br J Anaesth. 2010, 2:313. 10.1093/bja/aet114

8. Beard D, Harris K, Dawson J, Doll H, Murray D, Carr A, Price A: Meaningful changes for the Oxford hip and knee scores after joint replacement surgery. J Clin Epidemiol. 2015, 68:73-79. 10.1016/j.jclinepi.2014.08.009

9. Want D, Yang Y, Li Q, et al.: Adductor canal block versus femoral nerve block for total knee arthroplasty: a meta-analysis of randomized controlled trials. Sci Rep. 2017, 7:40721. 10.1038/srep40721

10. Andersen L, Kehlet H: Analgesic efficacy of local infiltration analgesia in hip and knee arthroplasty: a systematic review. Br J Anaesth. 2014, 113:360-374. 10.1093/bja/aeu155

11. Yun XD, Yin XL, Jiang J, Ting Y, Dong H, An L, Via Y: Local infiltration analgesia versus femoral nerve block in total knee arthroplasty: a meta-analysis. Orthop Traumatol Surg Res. 2015, 101:565-569. 10.1016/j.otsr.2015.03.015

12. Mei S, Jin S, Chen Z, Ding X, Zhao X, Li Q: Analgesia for total knee arthroplasty: a meta-analysis comparing 


\section{Cureus}

local infiltration and femoral nerve block. Clinics (San Paulo). 2015, 70:648-653. 10.6061/clinics/2015(09)09

13. Albrecht E, Guyen O, Jacot-Guillarmod A, Kirkham KR: The analgesic efficacy of local infiltration analgesia vs femoral nerve block after total knee arthroplasty: a systematic review and meta-analysis. Br J Anaesth. 2016, 116:597-609. 10.1093/bja/aew099

14. Ashraf A, Raut V, Canty S, McLauchlan G: Pain control after primary total knee replacement. A prospective randomised controlled trial of local infiltration versus single shot femoral nerve block. Knee. 2013, 20:324327. 10.1016/j.knee.2013.04.009

15. Fan L, Yu X, Zan P, Liu J, Ji T, Li G: Comparison of local infiltration analgesia with femoral nerve block for total knee arthroplasty: a prospective, randomized clinical trial. J Arthroplasty. 2016, 31:1361-1365. 10.1016/j.arth.2015.12.028

16. Moghtadaei M, Farahini H, Faiz S, Mokarami F, Safari S: Pain management for total knee arthroplasty: single-injection femoral nerve block versus local infiltration analgesia. Iran Red Crescent Med J. 2014, 16:13247. 10.5812/ircmj.13247

17. Parvataneni H, Shah V, Howard H, Cole N, Ranawat A, Ranawat C: Controlling pain after total hip and knee arthroplasty using a multimodal protocol with local periarticular injections: a prospective randomized study. J Arthroplasty. 2007, 22:33-38. 10.1016/j.arth.2007.03.034

18. Uesugi K, Kitano N, Kikuchi T, Sekiguchi M, Konno S: Comparison of peripheral nerve block with periarticular injection analgesia after total knee arthroplasty: a randomized study. Knee. 2014, 21:848-852. 10.1016/j.knee.2014.04.008 